\title{
OFDM Subcarrier Monitoring Using High Resolution Optical Spectrum Analysis
}

\author{
Josep M. Fabrega ${ }^{\mathrm{a}, *}$, Pascual Sevillano ${ }^{\mathrm{b}}$, Michela Svaluto Moreolo ${ }^{\mathrm{a}}$, \\ Asier Villafranca ${ }^{\mathrm{c}}$, F. Javier Vílchez ${ }^{\mathrm{a}}$, Jesús M. Subías ${ }^{\mathrm{b}}$ \\ ${ }^{a}$ CTTC, 08860, Castelldefels (Spain) \\ ${ }^{b}$ University of Zaragoza, 50018, Zaragoza (Spain) \\ ${ }^{c}$ Aragon Photonics Labs S.L., 50009, Zaragoza (Spain)
}

\begin{abstract}
In this paper, we demonstrate in-band OSNR monitoring of individual subcarriers in optical OFDM using a high-resolution optical spectrum analyzer. The relationships between OSNR, electrical SNR and BER at the receiver are experimentally analyzed and compared to theoretical results. A linear dependency in $\mathrm{dB}$ is found between electrical SNR and subcarrier OSNR for total OSNR values below $26 \mathrm{~dB}$. Above this limit, the correlation degree decreases due to the electrical SNR degradation at the edge subcarriers. The BER analysis per subcarrier also shows a clear correlation with the proposed in-band optical measurements.

Keywords: Optical spectrum analysis, optical performance monitoring, fiber optic communications.
\end{abstract}

\section{Introduction}

Optical performance monitoring of signal quality is one of the key enablers of intelligent optical networks [1]. Precisely, transmission impairments monitoring is needed at the nodes of the network, where add/drop, routing and groom-

5 ing functionalities are performed. Reconfigurable optical add-drop multiplexers and optical cross-connects are usually configured and managed by a control

\footnotetext{
${ }^{*}$ Corresponding author

Email address: jmfabrega@cttc.es (Josep M. Fabrega)
}

Preprint submitted to Optics Communications

December 18, 2014 
plane, which requires also in-band knowledge of the transmission impairments (e.g. noise, cross-talks, filtering effects, non-linearities, attenuation, dispersion, and component faults). The monitoring information is used by the control layer for configuring and optimizing optical paths, determining the causes of potential problems, setting up signal degradation alarms, preventing failures, and activating the corresponding resiliency mechanisms [2]. For example, the monitoring information can be disseminated by the control layer in order to compute spatial and spectral routes by means of impairment aware algorithms [3]. Then, a suitable network resources allocation and management can be performed. The optical signal to noise ratio (OSNR) is the most common parameter used to measure the degradation of signal the quality, because it is transparent to bit rate and modulation format, and can be easily related to the bit error ratio (BER), which is the main performance indicator [1].

The advent of new and advanced optical modulation formats is attractive for improving the transmission performance; even they pose new challenges and/or opportunities from the optical performance monitoring point of view. Precisely, optical orthogonal frequency division multiplexing (O-OFDM) has gained attention in optical communications as it represents a promising candidate for high data rate optical systems and enables software-defined optical transmission [4]. In fact, this modulation format is able to provide high spectral efficiency, robustness to chromatic dispersion and scalability to higher bit rates. Furthermore, this multicarrier-modulation is suitable for future elastic and adaptive optical networks $[4,5]$. In these networks, the bandwidth and bit rate of the OFDMbased transponders can be configured by the control layer, varying the electronic digital signal processing (DSP) of the transponder and, thus, properly selecting the number of subcarriers and the modulation format.

Additionally to the robustness against transmission impairments, O-OFDM can provide electronic dispersion compensation. The overhead of information

35 (e.g. pilot tones, training symbols, cyclic prefix), which must be allocated for correctly recovering the signal, allows monitoring system parameters for channel estimation and performance optimization $[4,6]$. However, this self-performance 
monitoring technique is performed in the electrical domain, requiring an optical receiver front-end. For an appropriate network management, a more simple and non-intrusive signal quality monitoring per subcarrier would be desirable at the nodes of the network. Thus, the control layer could dynamically reconfigure the parameters of each O-OFDM transponder in order to overcome the signal degradation for specific subcarriers without the need for optical to electrical conversion. This can be achieved by balancing the modulation format per subcarrier and/or adding/suppressing subcarriers, when transmitting along a specific lightpath.

Among the DSP-based O-OFDM options, those based on intensity modulation and direct detection (IM/DD) constitute a cost-effective solution for the implementation of bit-rate and bandwidth variable transponders. So, these subsystems can be used in networks designed for more cost-sensitive market segments [7].

In this paper, subcarrier OSNR measurements for IM/DD O-OFDM systems are demonstrated using high-resolution optical spectrum analysis. We extend our previous work [8] by developing a method for relating the optical domain measurements with fundamental figures of merit for IM/DD O-OFDM, such as the electrical/digital signal to noise ratio (SNR) and the BER, each of them at a subcarrier level. This method is experimentally evaluated for an IM/DD O-OFDM system based on the Hartley transform, corroborating the above mentioned relationships.

The remainder of the paper is organized as follows. Section 2 deals with the method for estimating the OSNR per subcarrier (SC-OSNR). Afterwards, in section 3, an experimental setup is detailed. Next, section 4 reports and discusses the results of the experiments. Finally, the main conclusions are drawn in section 5 . 
(a)

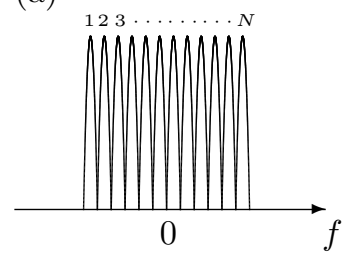

(b)

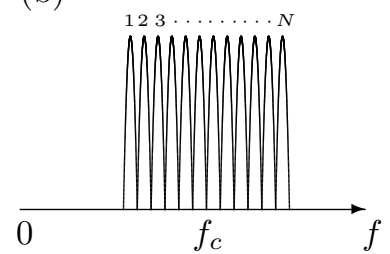

(c)

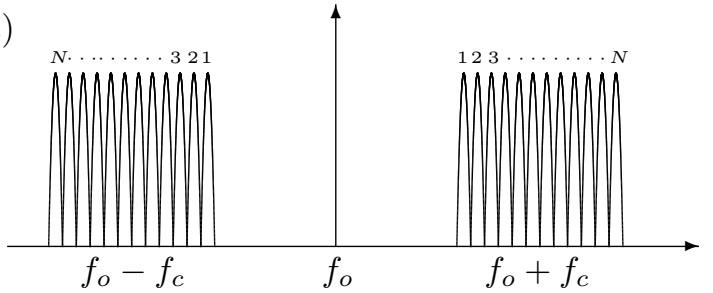

Figure 1: Spectra of an IM/DD O-OFDM signal: Baseband in the electrical domain (a), upconverted to $f_{c}(\mathrm{~b})$, and after modulation in the optical domain (c).

\section{OSNR Estimation Method}

The impact of the optical noise on each O-OFDM subcarrier can be assessed by a simple optical spectrum analysis. This allows obtaining an OSNR measurement in the optical domain, at the subcarrier level. However, some processing of the acquired optical spectrum samples must be performed in order to properly relate these measurements with the signals recovered at the receiver, in the digital domain.

A typical IM/DD O-OFDM power spectrum is depicted in Figure 1. Figure 1(a) shows the baseband electrical spectrum, before upconversion and optical modulation. As can be seen, the multiple subcarriers are centered in the origin of the frequency axis $(f=0)$. The OFDM subcarriers are numbered for the sake of clarity. As the signal is real-valued, the subcarriers feature an Hermitian symmetry with respect to 0 . Thus, according to the numbering in Figure 1(a), subcarrier 1 is the mirror-symmetric of subcarrier $N$, subcarrier 2 is the mirror-symmetric of subcarrier $N-1$, and so on.

For the case of real-valued fast Fourier transform (FFT), the symmetry of Figure 1(a) is forced at the DSP of the transmitter [9]. There, only half of the 


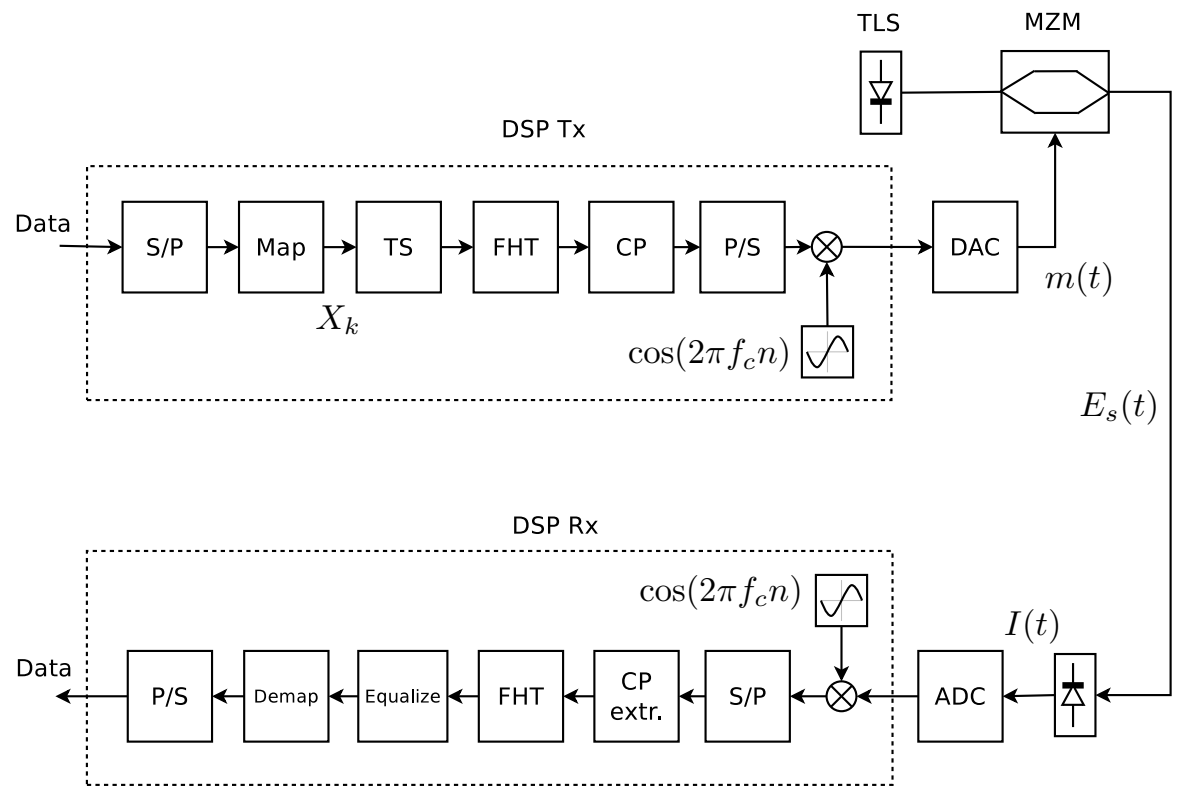

Figure 2: Simplified transmission scheme.

subcarriers support data (e.g. 1 through $N / 2$ ). The other half (e.g. $N / 2+1$ through $N$ ) carry the complex-conjugate of this data.

Alternatively, when the fast Hartley transform (FHT) is used for OFDM modulation, the spectral symmetry is obtained with no additional processing [10]. In the following lines, we will go through some concepts of the FHT, in order to detail how digital data are finally mapped onto the electrical and optical spectra following the transmission scheme depicted in Figure 2. At the transmitter DSP, input data are first parallelized and mapped onto a real constellation (e.g. binary phase shift keying - BPSK). Next, the training symbols (TS) are added and the transform is performed. It is worth noting that the FHT is a real transform with kernel [10]

$$
\cos \left(\frac{2 \pi k n}{N}\right)+\sin \left(\frac{2 \pi k n}{N}\right),
$$

being $N$ the total number of subcarriers, $n$ the number of sample, and $k=$ $1,2,3, \cdots, N$ the subcarrier number. An interesting property of the Hartley ${ }_{95}$ transform is that it is self-inverse [10] and, thus, the same implementation can 
be used at the transmitter and receiver signal processing.

After performing the transform, a cyclic prefix (CP) is added. Next, the resulting signal is serialized and upconverted to an intermediate frequency $f_{c}$. So, the upconverted OFDM signal after the digital to analog converter (DAC) can be described as

$$
m(t)=V_{\mathrm{DAC}} \cos \left(2 \pi f_{c} t\right) s(t),
$$

where $V_{\mathrm{DAC}}$ corresponds to the voltage swing present at the output of the DAC and $s(t)$ is the OFDM signal itself

$$
s(t)=\frac{1}{\sqrt{N}} \sum_{k=1}^{N} X_{k} T_{k}(t),
$$

with $X_{k}$ indicating the data symbol carried by the $k$-th subcarrier, and $T_{k}(t)$ standing for the $k$-th coefficient of the FHT

$$
T_{k}(t)=\cos \left(2 \pi f_{k} t\right)+\sin \left(2 \pi f_{k} t\right)
$$

being $t=n T$ and $f_{k}$ the $k$-th subcarrier frequency

$$
f_{k}=\frac{k}{T}-\frac{N}{2 T}
$$

where $T$ is the symbol period.

So, the optical field at the output of the Mach-Zehnder modulator can be expressed as

$$
E_{s}(t)=\sqrt{P_{s}} \cos \left(\frac{\pi}{2 V_{\pi}}\left(m(t)+V_{b}\right)\right) \exp \left(j 2 \pi f_{o} t\right),
$$

where $P_{s}$ is the optical power at the output of the modulator, $V_{b}$ is the bias voltage, $V_{\pi}$ is the switching voltage of the modulator, and $f_{o}$ is the optical carrier frequency.

In case the bias point of the Mach-Zehnder modulator is set to $V_{b}=-V_{\pi} / 2$ and assuming low voltage swing of $m(t)$, a pure intensity modulation is achieved and $\left|E_{s}(t)\right|^{2}$ can be approximated as

$$
\left|E_{s}(t)\right|^{2} \simeq \frac{P_{s}}{2}+\frac{P_{s}}{2} \frac{\pi}{V_{\pi}} m(t) .
$$


Figure 1(c) shows the double sideband optical spectrum after optical modulation. As expected, the electrical spectrum is symmetrically repeated at both sides of the optical frequency $f_{o}$.

At the receiver, a standard photodiode with responsivity $R$ is used, giving as output current

$$
I(t)=R \frac{P_{s}}{2}+R \frac{P_{s}}{2} \frac{\pi}{V_{\pi}} m(t)+n(t),
$$
being $n(t)$ a noise process, mainly contributed by optical noise.

After photodetection, the signal $I(t)$ is converted into the digital domain by an analog to digital converter (ADC). The resulting digital signal is processed in the DSP following the equivalent steps made in transmission, but in reverse order.

As expected, the digital OFDM signal is linearly mapped into the optical power, and can be recovered after photodetection with no distortion. Thus, if a direct spectrum analysis is made in the optical domain (prior to detection), the status of the OFDM signal carriers can be conveniently monitored.

The digitization, downconversion and FHT processing can be considered as a correlator bank plus sampling. Thus, after some algebra, the demodulated signal can be written as

$$
Q_{k}=\frac{1}{T} \int_{0}^{T}\left[\frac{\pi R P_{s} V_{\mathrm{DAC}}}{4 V_{\pi}} s(t)+n(t)\right] T_{k}(t) d t=S_{k}+N_{k}
$$

$S_{k}$ is the undistorted signal and $N_{k}$ is the noise term. Similarly to [11], $S_{k}$ can be found as

$$
S_{k}=\frac{1}{T} \int_{0}^{T} \frac{\pi R P_{s} V_{\mathrm{DAC}}}{4 V_{\pi}} s(t) T_{k}(t) d t=\frac{\pi R P_{s} V_{\mathrm{DAC}}}{4 V_{\pi}} X_{k}
$$

and its power can be written as

$$
P_{x}=\frac{\pi^{2} R^{2} P_{s}^{2} V_{\mathrm{DAC}}^{2}}{4 V_{\pi}^{2} N} .
$$

Now, some assumptions on the noise should be considered. When the signal $E_{s}(t)$ is degraded by some optical noise (e.g. due to optical amplification), 
a certain SNR degradation is obtained after photodetection. According to the noise beating theory [12], the beating between signal and noise can be considered dominant for high OSNR, and it can be approximated as Gaussian noise. Thus, the power spectrum of $n(t)$ is approximately flat and with density

$$
G_{n}(f)=\frac{2 R^{2} P_{s}^{2}}{\mathrm{SNR}_{o} B_{o}}
$$

where $B_{o}$ is the optical reference bandwidth for measuring the OSNR, and $\mathrm{SNR}_{o}=P_{s} / P_{n o}$ is the global optical OSNR, being $P_{n o}$ the power of optical noise within $B_{o}$.

According to (4) and (9), $N_{k}$ can be expressed as two Fourier coefficients of $n(t)$ evaluated at $f=f_{k} / 2$, whose variance is proportional to $G_{n}(f)$ evaluated at $f=f_{k} / 2[11]$. After making the necessary calculations, the variance of $N_{k}$ can be expressed as

$$
\sigma_{N}^{2}=\frac{1}{4} \frac{1}{2 T} \frac{2 R^{2} P_{s}^{2}}{\operatorname{SNR}_{o} B_{o}} .
$$

The output of the $k$-th correlator is thus comprised of a signal component $S_{k}$ and an additive Gaussian noise component $N_{k}$, leaving an SNR of

$$
\mathrm{SNR}_{e}=\frac{P_{x}}{\sigma_{N}^{2}}=\frac{\pi^{2} V_{\mathrm{DAC}}^{2} B_{o}}{V_{\pi}^{2} N B_{e}} \mathrm{SNR}_{o}
$$

150

being $B_{e}=1 / T$ the equivalent bandwidth occupied by each OFDM subcarrier.

So the probability of error can be found as that of BPSK modulated data with additive Gaussian noise [13]

$$
P_{e}=\frac{1}{2} \operatorname{erfc} \sqrt{\frac{\mathrm{SNR}_{e}}{2}} .
$$

Since a high resolution optical spectrum analysis is to be performed, an OSNR figure per subcarrier is defined. For convenience, this subcarrier OSNR 155 subcarrier $P_{s c}$, and the power of the optical noise $P_{n o}$. Using (2), (3), and (7), $P_{s c}$ can be written as

$$
P_{s c}=\frac{1}{2} \frac{\pi V_{\mathrm{DAC}}}{V_{\pi} \sqrt{N}} P_{s}
$$


consequently, the OSNR per subcarrier is

$$
\mathrm{SNR}_{o s c}=\frac{P_{s c}}{P_{n o}}=\frac{\pi V_{\mathrm{DAC}}}{V_{\pi} \sqrt{N}} \mathrm{SNR}_{o} .
$$

Thus, a linear relationship between $\mathrm{SNR}_{o s c}$ and $\mathrm{SNR}_{e}$ can be found as

$$
\frac{\mathrm{SNR}_{o s c}}{\mathrm{SNR}_{e}}=\frac{1}{2} \frac{V_{\pi} \sqrt{N}}{\pi V_{\mathrm{DAC}}} \frac{B_{e}}{B_{o}} .
$$

by Figure $1(\mathrm{~b})$, the symmetry is with respect to $f_{c}$. Therefore, the FHT is an Hermitian linear operator, similarly to the real-valued FFT.

In order to assess the impact of the optical signal degradation at a subcarrier level, the optical spectrum of Figure 1(c) has to be mapped with the 
of Figure 1(a) and Figure 1(b). The procedure to employ is the following

1. A SC-OSNR is measured in the spectral interval occupied by each subcarrier. In contrast with the total OSNR defined for $0.1 \mathrm{~nm}$ and accounting for an average performance of the whole system, this value allows specific characterization and performance degradation monitoring for each subcarrier.

2. The SC-OSNR contributions at the two sides of the optical carrier are averaged in order to take into account the symmetry due to the optical modulation. At this point, we obtain an SC-OSNR spectral distribution like Figure 1(b) from Figure 1(c).

3. In order to take into account the inherent Hermitian symmetry of the FHT (or a real-valued FFT), a further average of the SC-OSNR is performed between both sides of each OFDM band.

The resulting averaged SC-OSNR is a figure of merit which takes into account all the symmetries of the double sideband IM/DD spectra and, thus, can be directly related to the figures obtained in the digital domain, after OFDM demodulation. Additionally, this methodology can be easily adapted to other OFDM techniques. For example, when data is mapped into a complex-valued FFT combined with I/Q modulation and coherent detection, a more straightforward mapping between optical spectral components and data symbols is present, and step 3 can be avoided.

\section{Experimental Setup}

In order to perform a first test of the OSNR monitoring at subcarrier level, the experimental set-up described in Figure 3 is used for a back-to-back configuration. It is conceived to characterize the correlation between the different levels of OSNR values in the optical domain and the performance analysis parameters in the electrical domain. The DSP and electrical up/down-conversion at the transmitter/receiver are performed off-line using Matlab, following the 
steps detailed in Figure 2. At the transmitter, data are pseudo-randomly generated using the Mersenne-Twister generator [14]. Next, these data are mapped into the corresponding constellation (BPSK) and modulated by an FHT, with 64 subcarriers. The system is intended for occupying a $12.5 \mathrm{GHz}$ channel, as it is the minimum slot width envisioned in an elastic and flexible networking environment [15]. Consequently, the total bandwidth of the generated optical spectrum is set to $11 \mathrm{GHz}$. A small guard band of $500 \mathrm{MHz}$ around the optical carrier $f_{o}=193.865 \mathrm{THz}(1550.92 \mathrm{~nm})$ is set for avoiding undesired effects from the laser emission profile. Thus, the considered electrical bandwidth occupancy for the useful OFDM signal ( 51 subcarriers) is $5 \mathrm{GHz}$. The bit rate is $5 \mathrm{~Gb} / \mathrm{s}$, as a BPSK modulation format is used. The obtained real-valued OFDM symbols are then serialized. Every 2048 OFDM frames, 8 training symbols are inserted for synchronization and equalization. The digital OFDM signal is clipped and upconverted to an intermediate frequency $\left(f_{c}=3 \mathrm{GHz}\right)$ by mixing it with a digital oscillator. The resulting signal is converted to the analog domain by an arbitrary waveform generator (AWG) running at $12 \mathrm{GSa} / \mathrm{s}$. This analog signal is conditioned and injected to a Mach-Zehnder modulator (MZM) biased at the quadrature point and excited by a tunable laser source (TLS) operating at $1550.92 \mathrm{~nm}$.

In order to experimentally analyze the optical noise performance, an additive white noise generated by an Erbium doped fiber amplifier (EDFA), followed by 230 a variable optical attenuator (VOA), is introduced in the system by means of a 50:50 coupler. An additional EDFA is placed after the coupler for ensuring enough optical power for the spectral analysis and when employing the optical receiver. In order to relax the working conditions of this EDFA, an optical filter can be placed between the VOA and the 50:50 coupler.

The spectral analysis is performed with a high resolution optical spectrum analyzer (Aragon Photonics, BOSA 200). The equipment achieves $10 \mathrm{MHz}$ resolution based on the use of stimulated Brillouin scattering (SBS) as an active optical filter [16]. Compared with other filtering techniques the main advantage of SBS is the high optical rejection ratio that it can achieve. Due to its non- 


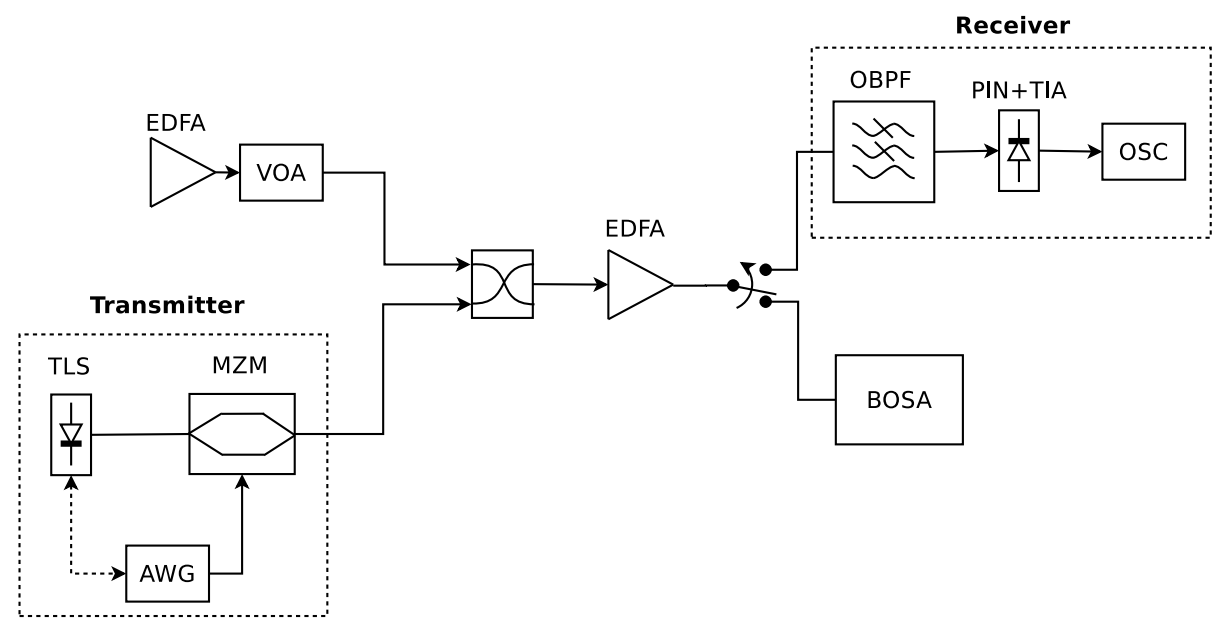

Figure 3: Experimental setup. demapped according to Figure 2. 


\section{Results and Discussion}

Several OSNR values are analysed, ranging from $18 \mathrm{~dB}$ up to $32 \mathrm{~dB}$, within

260 order to distinguish them from SC-OSNR. Sample optical spectra are depicted in Figure 4 using a resolution bandwidth of $10 \mathrm{MHz}$ at $-3 \mathrm{~dB}$. Please note that for the case of $28 \mathrm{~dB}$ all the useful signal within the OFDM bands is above the optical noise, as shown in Figure 4(a). For the case of $18 \mathrm{~dB}$ OSNR, part of the the OSNR dependency over the FHT subcarriers. There it can be observed 

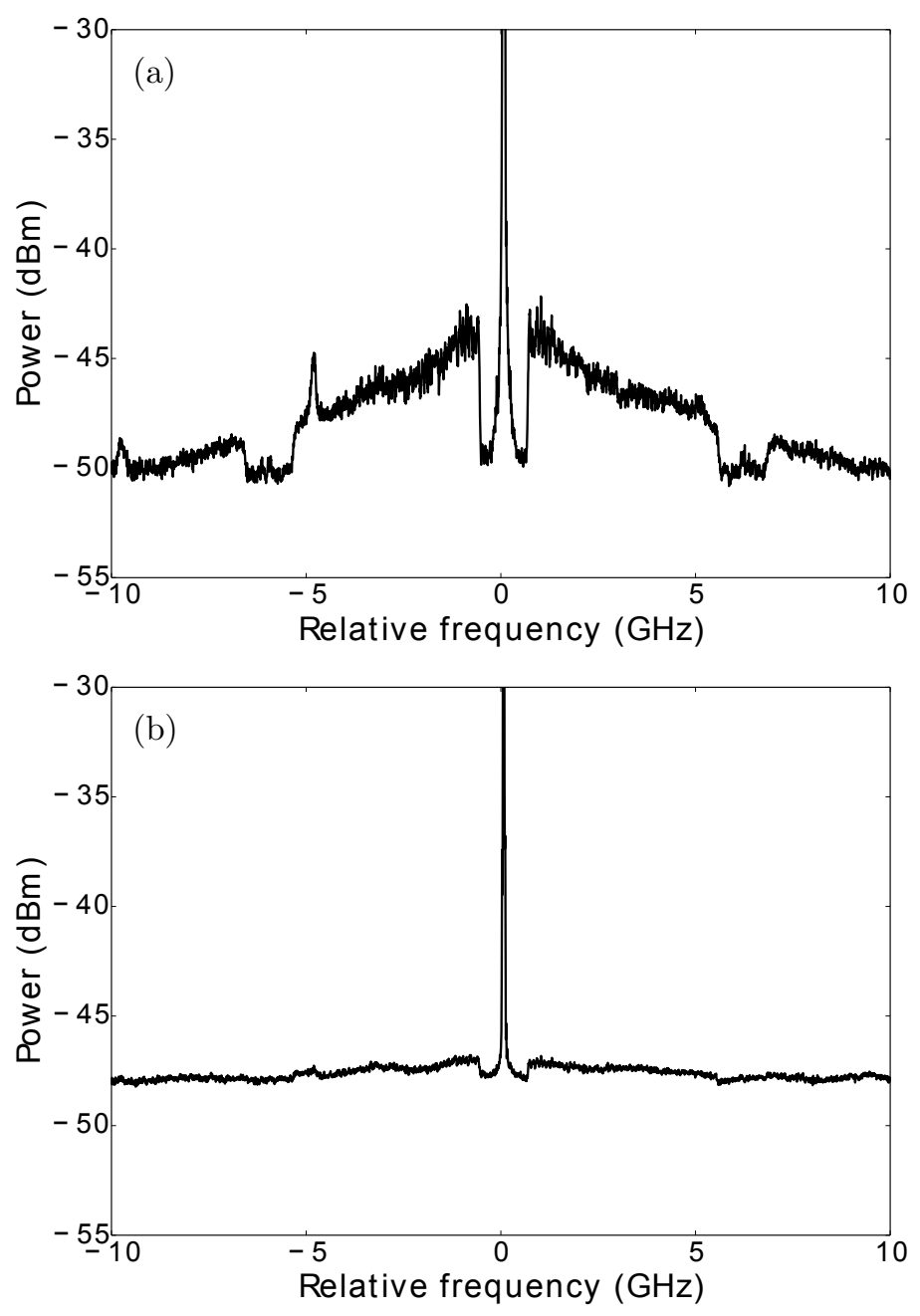

Figure 4: Optical spectrum before the receiver with (a) $28 \mathrm{~dB}$ OSNR and (b) $18 \mathrm{~dB}$ OSNR. 


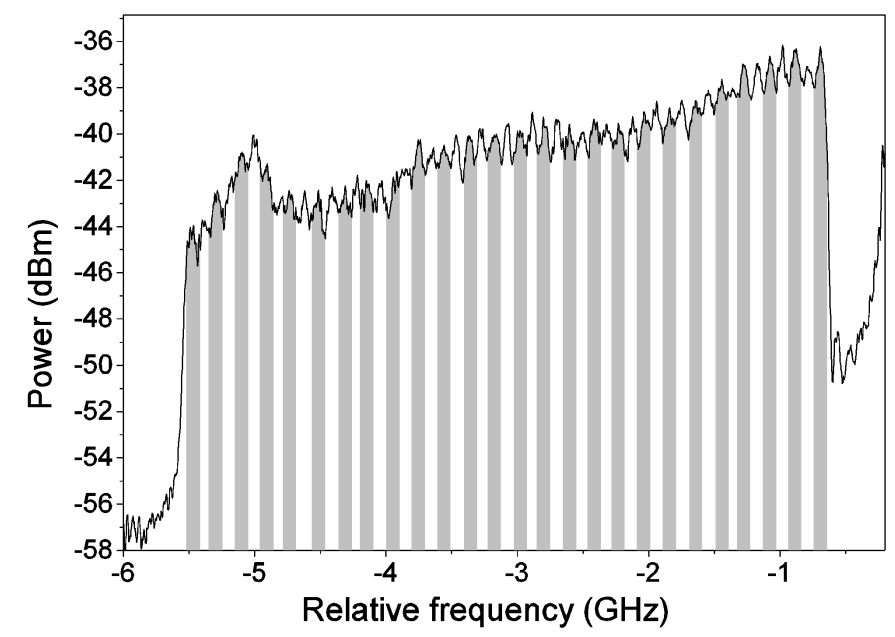

Figure 5: Sample optical spectrum of one of the modulation sidebands. The bandwidth of the different subcarriers is evidenced alternating white and grey colors.

the OSNR dependency over the FHT subcarriers. Furthermore, the subcarriers that are more affected by the optical noise can be easily identified. For example, the first and last sub-sets of subcarriers (neighborhoods of the 10-th and 53-th subcarriers) have a value of OSNR greater than $3 \mathrm{~dB}$ compared to the central subcarriers.

Similarly, the signal at the receiver is recovered just after the FHT block, where the electrical SNR is calculated as the quotient between the mean power and the variance per subcarrier, for the proposed range of total OSNR values. The distribution of this electrical SNR over the FHT subcarriers is depicted in Figure 6(b). There it can be seen how the subcarriers are affected by the noise, after photodetection and DSP. For total OSNR values of $26 \mathrm{~dB}$ and below, the subcarriers in the neighborhood of 10-th and 53-rd subcarriers are less affected by the noise than the central ones (about $2 \mathrm{~dB}$ difference). Nevertheless, above $26 \mathrm{~dB}$ total OSNR, the central subcarriers outperform the ones at the edges. This is due to synchronization errors, mainly contributed by a small sampling frequency offset between transmitter and receiver, whose effects are only noticeable for high values of total OSNR. Please note that the impact of 

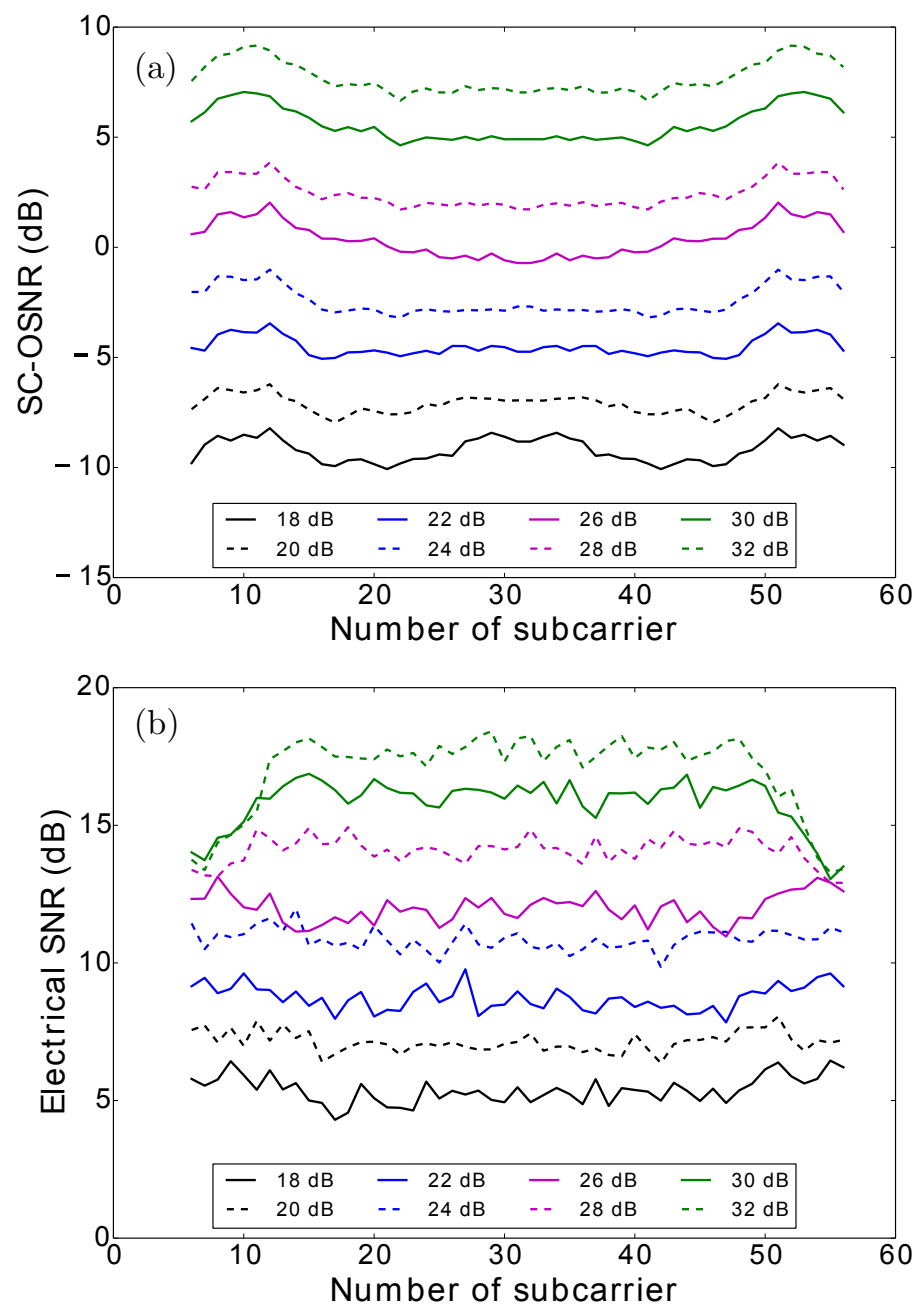

Figure 6: (a) Distribution of the SC-OSNR from the measurement of the optical spectrum, per subcarrier. (b) Distribution of the electrical SNR measured before equalizing, per subcarrier.

the sampling frequency offset depends on the number of subcarrier, degrading more the edges of the OFDM band than the central subcarriers [18].

A direct relationship is found between the SC-OSNR calculated from the high-resolution OSA spectrum and the electrical SNR calculated at the receiver. This is shown in Figure 7, where the SC-OSNR is plotted as a function of the electrical SNR before equalization (a) and after equalization (b). As expected, 
the electrical SNR before equalization is highly correlated with the SC-OSNR. In fact, their dependency is almost linear in $\mathrm{dB}$, meaning that increasing one $\mathrm{dB}$ of electrical SNR is directly translated to a $1 \mathrm{~dB}$ increase of SC-OSNR. Also, for electrical SNR in the neighborhood of $15 \mathrm{~dB}$ and higher, the theoretical model based on (18) is a lower limit, following the curve trend. For values of electrical SNR above $13 \mathrm{~dB}$, there is a high dispersion of points, due to the fact observed in Figure 6(b). For total OSNR values beyond $26 \mathrm{~dB}$, the electrical SNR of the subcarriers at the edges is limited to $\sim 13 \mathrm{~dB}$. In order to overcome this effect, the electrical SNR is calculated after equalization, as shown in Figure 7(b). For this case, the points corresponding to higher electrical SNR have lower dispersion. However, the other parts of the plot show less correlation between SC-OSNR and electrical SNR.

Finally, BER measurements are obtained by statistical bit error counting up to obtain $10^{3}$ errors. Results are shown in Figs. 8, 9, and 10. Figure 8 shows the total BER as a function of the total OSNR. There it can be observed that $10^{-3}$ BER is attained at $23.7 \mathrm{~dB}$ of OSNR.

Figure 9 shows the BER distribution over the active OFDM subcarriers at different values of total OSNR, for a single acquisition of $2^{15}$ bits. Comparing Figure 6 with Figure 9, it can be seen a clear correlation between both, as expected. For example, in the neighborhood of the 10-th and 53-rd subcarriers, the BER is much lower than for the central ones (about one order of magnitude).

Also a direct relationship is found between SC-OSNR measured with BOSA and BER. This is shown in Figure 10. As expected, the BER is correlated with the SC-OSNR, in a similar way to the total OSNR, see Figure 6(a), decreasing one order of magnitude per $5 \mathrm{~dB}$ increase of SC-OSNR. Also, the BER calculated employing (18), represented with a continuous line, acts as an upper limit. This is because the theoretical model describes the limit performance, when SC-OSNR is above $15 \mathrm{~dB}$ and assuming a Gaussian distribution of the electrical noise, according to the discussion presented in section 2 . 

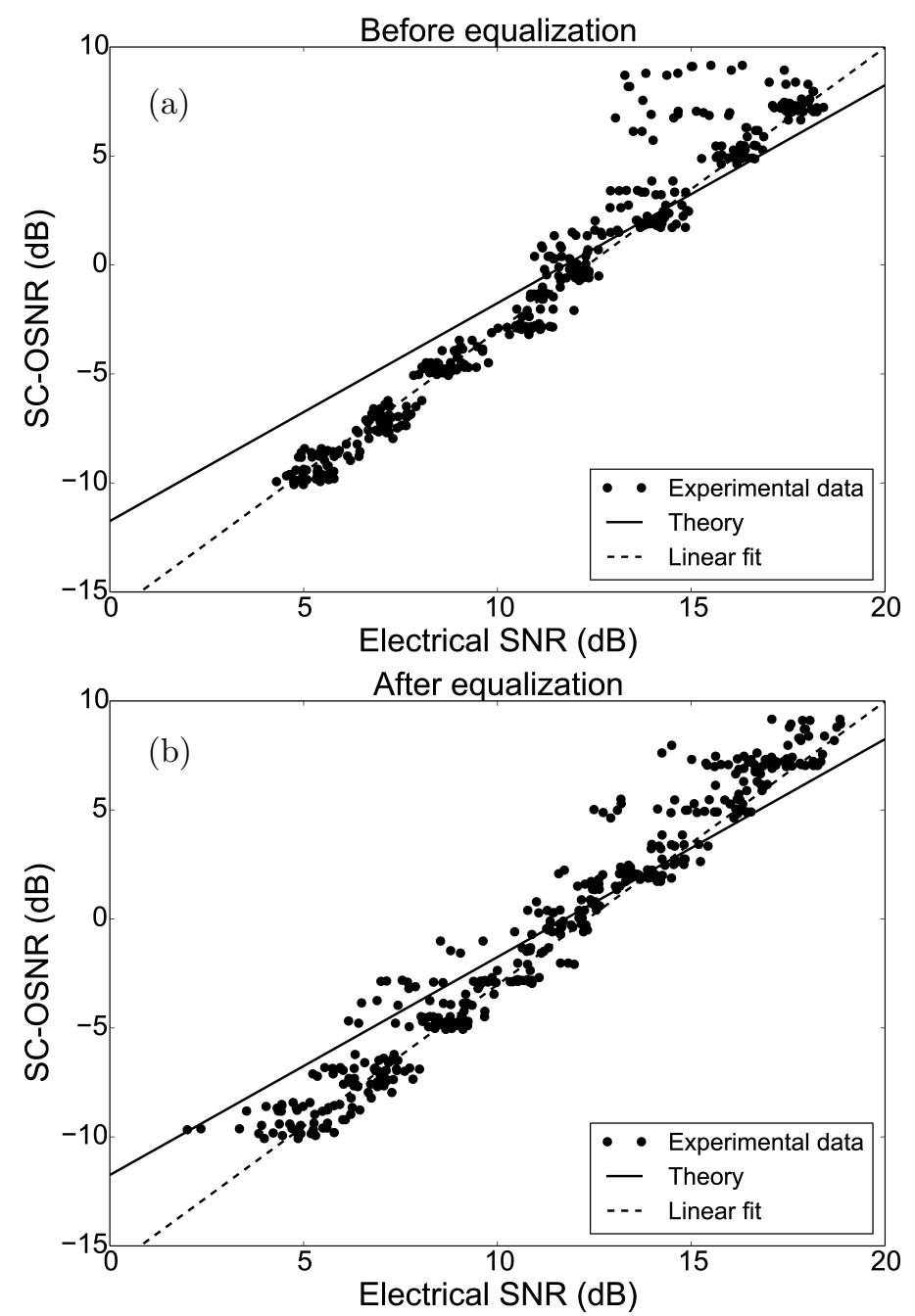

Figure 7: SC-OSNR as function of the electrical SNR taken (a) before equalization and (b) after equalization. Points are the values for different subcarriers and at different total OSNR values. Continuous line corresponds to theoretical model based on equation (18). Dashed line is the linear fit (in $\mathrm{dB}$ ) of the points.

\section{Conclusion}

In this work we have proposed a methodology to estimate the sub-carrier OSNR and we have demonstrated the direct correlation between the BER performance of individual sub-carriers and the measured sub-carrier OSNR. Live 


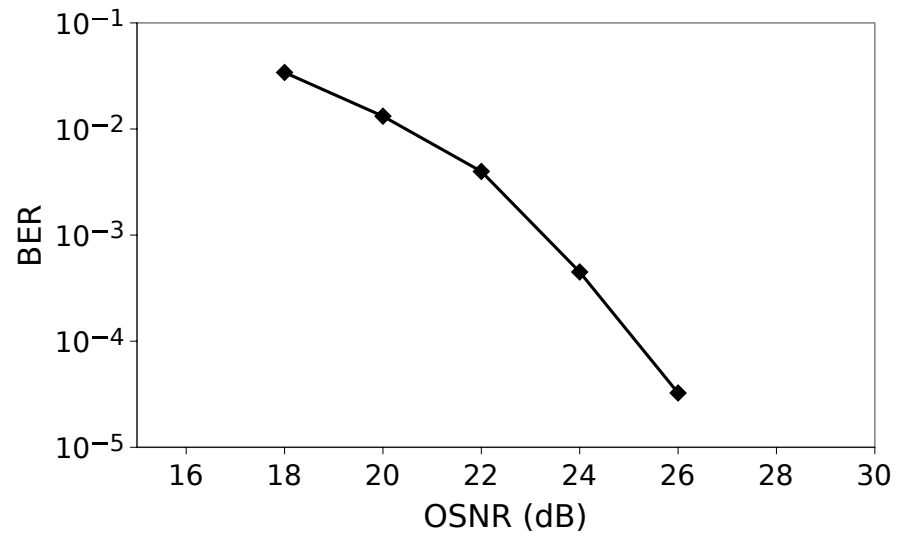

Figure 8: Total BER as function of the total OSNR.

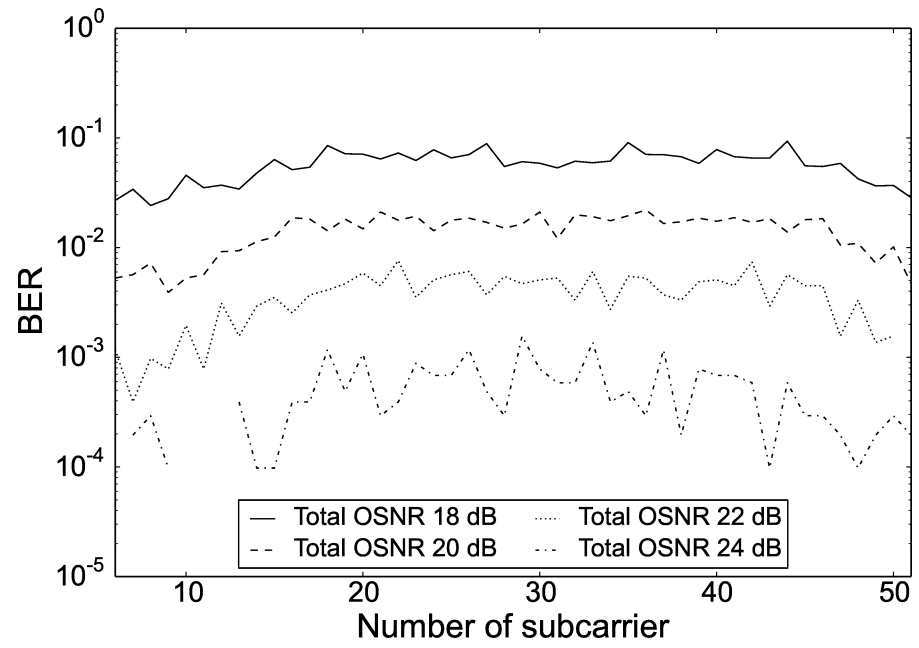

Figure 9: Distribution of BER per OFDM subcarrier.

monitoring of these quality parameters can be performed at the nodes of the optical networks with no need for demodulating data. Thus, it becomes a key point towards better management of OFDM-based systems in future flexible and elastic optical networks. Additionally, the methodology developed can be easily adapted to be used when employing other OFDM techniques, as coherent optical OFDM. 


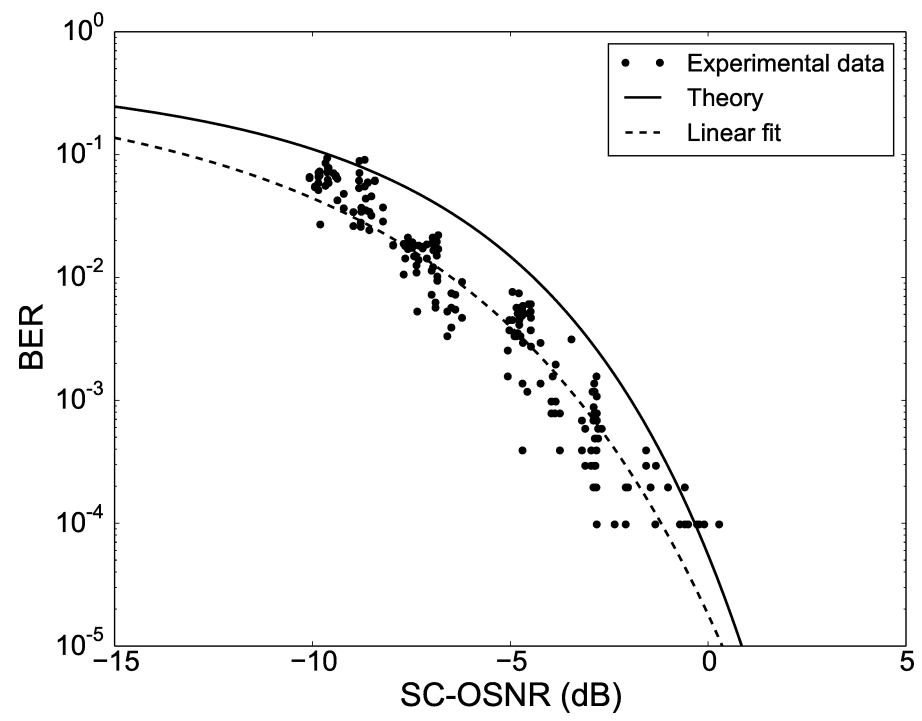

Figure 10: BER as function of the SC-OSNR. Points correspond to values for different subcarriers and at different total OSNR values.

\section{Acknowledgements}

This work was supported by EU/FP7 through project IDEALIST (G.A. 317999), MICYT through Project TSI-020100-2011-423 and by MINECO through projects TEC2010-17869 and TEC2012-38119 (FARO)

\section{References}

[1] C. C. K. Chan, Optical Performance Monitoring Techniques, Elsevier, 2010.

[2] R. Ramaswami, K. Sivarajan, G. Sasaki, Optical Networks: a Practical Perspective, Morgan Kaufmann, 2009.

[3] R. Martínez, R. Casellas, R. Munoz, T. Tsuritani, Experimental Translucent-Oriented Routing for Dynamic Lightpath Provisioning in GMPLS-Enabled Wavelength Switched Optical Networks, J. Lightwave Technol. 28 (8) (2010) 1241-1255. doi:10.1109/JLT.2010.2043335.

[4] W. Shieh, I. Djordjevic, Orthogonal Frequency Division Multiplexing for Optical Communications, Elsevier, 2010. 
[5] M. Jinno, H. Takara, B. Kozicki, Y. Tsukishima, Y. Sone, S. Matsuoka,

[10] R. N. Bracewell, The Fourier Transform and its Applications, McGraw-Hill New York, 2000.

[11] J. M. Fabrega, M. Svaluto Moreolo, M. Chochol, G. Junyent, Impact of Modulator Driving on Constant Envelope Optical OFDM Based on Hartley Spectrum-Efficient and Scalable Elastic Optical Path Network: Architecture, Benefits, and Enabling Technologies, Communications Magazine, IEEE 47 (11) (2009) 66-73. doi:10.1109/MCOM.2009.5307468.

[6] M. Svaluto Moreolo, J. M. Fabrega, L. Nadal, F. Vilchez, Software-Defined Optical OFDM Transmission Systems: Enabling Elasticity in the Data Plane, in: 14th International Conference on Transparent Optical Networks (ICTON), 2012, 2012, pp. 1-4. doi:10.1109/ICTON.2012.6254504.

[7] M. Svaluto Moreolo, J. M. Fabrega, L. Nadal, F. Vilchez, V. Lopez, J. Fernandez-Palacios, Cost-effective data plane solutions based on OFDM technology for flexi-grid metro networks using sliceable bandwidth variable transponders, in: Optical Network Design and Modeling, 2014 International Conference on, 2014, pp. 281-286.

[8] J. M. Fabrega, P. Sevillano, M. Svaluto Moreolo, J. J. Martinez, A. Villafranca, J. Subias, All-optical in-band OSNR measurement in intensitymodulated direct-detection optical OFDM systems, in: 15th International Conference on Transparent Optical Networks (ICTON), 2013, 2013, pp. 1-4. doi:10.1109/ICTON.2013.6602731.

[9] B. Schmidt, A. Lowery, J. Armstrong, Experimental Demonstrations of Electronic Dispersion Compensation for Long-Haul Transmission Using Direct-Detection Optical OFDM, Lightwave Technology, Journal of 26 (1) (2008) 196-203. doi:10.1109/JLT.2007.913017. Transform, Photonics Technology Letters, IEEE 25 (6) (2013) 598-601. doi:10.1109/LPT.2013.2246785. 
[12] G. P. Agrawal, Fiber-Optic Communication Systems, 3rd Edition, John Wiley \& Sons, 2002.

[13] J. G. Proakis, Digital Communications, McGraw Hill, 1989.

[14] M. Matsumoto, T. Nishimura, Mersenne Twister: A 623-dimensionally Equidistributed Uniform Pseudo-random Number Generator, Transactions on Modeling and Computer Simulation, ACM 8 (1) (1998) 3-30. doi:10.1145/272991.272995.

[15] ITU-T Recommendation G.694.1: Spectral grids for WDM applications: DWDM frequency grid, Tech. rep., International Telecommunication Union (Feb 2012).

[16] J. M. Subias, J. Pelayo, F. Villuendas, C. Heras, E. Pellejer, Very High Resolution Optical Spectrometry by Stimulated Brillouin Scattering, Photonics Technology Letters, IEEE 17 (4) (2005) 855-857. doi:10.1109/LPT . 2005.843946.

[17] D. Baney, B. Szafraniec, A. Motamedi, Coherent Optical Spectrum Analyzer, Photonics Technology Letters, IEEE 14 (3) (2002) 355-357. doi:10.1109/68.986811.

[18] M. Sliskovic, Carrier and Sampling Frequency Offset Estimation and Cor410 rection in Multicarrier Systems, in: Global Telecommunications Conference, 2001. GLOBECOM '01. IEEE, Vol. 1, 2001, pp. 285-289 vol.1. doi:10.1109/GLOCOM.2001.965124. 